\title{
A Canonical Quadratic Form on the Determinant Line of a Flat Vector Bundle
}

\author{
Maxim Braverman ${ }^{1}$ and Thomas Kappeler ${ }^{2}$ \\ ${ }^{1}$ Department of Mathematics, Northeastern University, Boston, MA \\ 02115, USA and ${ }^{2}$ Institut fur Mathematik, Universitat Zürich, \\ Winterthurerstrasse 190, CH-8057 Zürich, Switzerland
}

Correspondence to be sent to: maximbraverman@neu.edu

We introduce and study a canonical quadratic form, called the torsion quadratic form, on the determinant line of the cohomology of a flat vector bundle over a closed oriented odd-dimensional manifold. This quadratic form caries less information than the refined analytic torsion, introduced in our previous work, but is easier to construct and closer related to the combinatorial Farber-Turaev torsion. In fact, the torsion quadratic form can be viewed as an analytic analogue of the Poincaré-Reidemeister scalar product, introduced by Farber and Turaev. Moreover, it is also closely related to the complex analytic torsion defined by Cappell and Miller and we establish the precise relationship between the two. In addition, we show that up to an explicit factor, which depends on the Euler structure, and a sign the Burghelea-Haller complex analytic torsion, whenever it is defined, is equal to our quadratic form. We conjecture a formula for the value of the torsion quadratic form at the Farber-Turaev torsion and prove some weak version of this conjecture. As an application, we establish a relationship between the Cappell-Miller and the combinatorial torsions.

\section{Introduction}

In [5], we constructed a new invariant of a flat vector bundle $(E, \nabla)$ over a closed oriented manifold $M$ of odd dimension $d=2 r-1$. It is a quadratic form $\tau=\tau_{\nabla}$, called the torsion 
quadratic form, on the determinant line $\operatorname{Det}\left(H^{\bullet}(M, E)\right)$ of the cohomology of $E$, which we defined in terms of another, more sophisticated invariant, the refined analytic torsion $\rho_{\text {an }} \in \operatorname{Det}\left(H^{\bullet}(M, E)\right)$, constructed in [6-8].

The invariant $\tau$ is closely related to the quadratic form $\tau^{\mathrm{BH}}=\tau_{\nabla, b}^{\mathrm{BH}}$, introduced by Burghelea and Haller [11]. To construct $\tau^{\mathrm{BH}}$ they need to require that the bundle $E$ admits a complex-valued nondegenerate bilinear form $b$. The definition of $\tau^{\mathrm{BH}}$ is similar to the definition of the Ray-Singer torsion, but instead of the standard Laplacians on differential forms uses the non-self-adjoint Laplace-type operators $\Delta_{b}=$ $\nabla \nabla_{b}^{\#}+\nabla_{b}^{\#} \nabla$, where $\nabla_{b}^{\#}$ denotes the adjoint of $\nabla$ with respect to the bilinear form $b$. Recall that the Ray-Singer torsion is a combination of the square roots of the determinants of the standard Laplacians. Since the determinants of the non-self-adjoint operators $\Delta_{b}$ are complex numbers their square roots are not canonically defined. This is the reason why Burghelea and Haller defined $\tau^{\mathrm{BH}}$ in terms of the determinants of $\Delta_{b}$ rather than their square roots, extending in this way the square of the Ray-Singer torsion.

Farber and Turaev $[18,19]$ defined a combinatorial torsion $\rho_{\varepsilon, 0} \in \operatorname{Det}\left(H^{\bullet}(M, E)\right)$, which depends on the orientation $\mathfrak{o}$ of the cohomology $H^{\bullet}(M)$ and on the Euler structure $\varepsilon$ introduced by Turaev [27, 28]. It was noticed by Burghelea [9] that the Euler structure $\varepsilon$ can be described by a closed form $\alpha_{\varepsilon} \in \Omega^{d-1}(M)$. Extending the classical Ray-Singer conjecture, $[4,15,23,24]$, Burghelea and Haller conjectured that

$$
\tau_{\nabla, b}^{\mathrm{BH}}\left(\rho_{\varepsilon, 0}\right)=e^{\int_{M} \omega_{\nabla, b} \wedge \alpha_{\varepsilon}},
$$

where $\omega_{\nabla, b}=-\frac{1}{2} \operatorname{Tr} b^{-1} \nabla b$ is the Kamber-Tondeur form, which measures the nonflatness of the bilinear form $b$. This conjecture was proven up to sign by Burghelea-Haller [10] and in full generality by Su-Zhang [26].

In [5], we showed that $\tau^{\mathrm{BH}}= \pm \tau$ whenever $\tau^{\mathrm{BH}}$ is defined and extended the Burghelea-Haller conjecture to the case when $\tau^{\mathrm{BH}}$ is not defined. More precisely, we conjectured, cf. [5, Conjecture 1.12], that

$$
\tau_{\nabla}\left(\rho_{\varepsilon, 0}\right)=e^{2 \pi i\left\langle\operatorname{Arg}_{\nabla}, c(\varepsilon)\right\rangle}
$$

Here $c(\varepsilon) \in H_{1}(M, \mathbb{Z})$ is the characteristic class of the Euler structure $\varepsilon$, cf. [28, Section 5.3]; $\operatorname{Arg}_{\nabla} \in H^{1}(M, \mathbb{C} / \mathbb{Z})$ is the unique cohomology class such that for every closed curve $\gamma$ in $M$ we have

$$
\operatorname{det}\left(\operatorname{Mon}_{\nabla}(\gamma)\right)=\exp \left(2 \pi i\left\langle\operatorname{Arg}_{\nabla},[\gamma]\right\rangle\right)
$$


where $\operatorname{Mon}_{\nabla}(\gamma)$ denotes the monodromy of the flat connection $\nabla$ along the curve $\gamma$; finally, $\langle\cdot, \cdot\rangle$ denotes the natural pairing $H^{1}(M, \mathbb{C} / \mathbb{Z}) \times H_{1}(M, \mathbb{Z}) \rightarrow \mathbb{C} / \mathbb{Z}$.

Note that (1.1) implies (1.2) whenever $\tau^{\mathrm{BH}}$ is defined, see [5, Section 1.11]. In [5] we proved the following weak version of Conjecture (1.2): For each connected component $\mathcal{C}$ of the space of flat connections on $E$ there exists a constant $R_{\mathcal{C}} \in \mathbb{C}$ with $\left|R_{\mathcal{C}}\right|=1$, such that

$$
\tau_{\nabla}\left(\rho_{\varepsilon, \mathfrak{o}}\right)=R_{\mathcal{C}} \cdot e^{2 \pi i\left\langle\operatorname{Arg}_{\nabla}, c(\varepsilon)\right\rangle} .
$$

Farber and Turaev [19, Section 9] introduced a bilinear form $\langle\cdot, \cdot\rangle_{\mathrm{PR}}$ on $\operatorname{Det}\left(H^{\bullet}(M, E)\right)$, which they call the cohomological Poincaré-Reidemeister scalar product. This is an invariant that refines the Poincaré-Reidemeister metric introduced by Farber [16]. It follows from Theorem 9.4 of [19] that Conjecture (1.2) is equivalent to the statement that

$$
\tau_{\nabla}(\cdot)=(-1)^{z}\langle\cdot, \cdot\rangle_{\mathrm{PR}},
$$

where $z \in \mathbb{N}$ is defined in formula (6.5) of [19].

Another related invariant $T \in \operatorname{Det}\left(H^{\bullet}(M, E)\right) \otimes \operatorname{Det}\left(H^{\bullet}(M, E)\right)$ was introduced by Cappell and Miller [14]. To define $T$ they also used non-self-adjoint Laplace-type operators, but different from the ones used by Burghelea and Haller. In fact, they consider the square $\mathcal{B}^{2}$ of the Atiyah-Patodi-Singer odd signature operator $\mathcal{B}=\mathcal{B}\left(\nabla, g^{M}\right)$ and, hence, don't need any additional assumptions on E. Further in [14], Cappell and Miller conjectured that, in an appropriate sense, their torsion is equal to the Reidemeister torsion of the bundle $E \oplus E^{*}$, where $E^{*}$ denotes the dual bundle to $E$.

The goal of this paper is to present a simple construction of the torsion quadratic form $\tau$, implicitly already contained in [7]. We collect only those parts of [5, 7, 8], which are needed for this purpose. In particular, we bypass the refined analytic torsion. Recall that the definition of the refined analytic torsion in $[7,8]$ uses the graded determinant of the odd signature operator $\mathcal{B}$, leading to a rather complicated analysis, involving the determinant of $\mathcal{B}^{2}$ and the $\eta$-invariant. In contrast, the definition of $\tau$ only involves the determinant of the Laplace-type operator $\mathcal{B}^{2}$. It turns out that the construction of $T$ by Cappell and Miller is very similar to our construction of $\tau$, as it uses the same operator $\mathcal{B}^{2}$. We establish the precise relationship of $T$ with $\tau$. It turns out that $T$ is the dual of $\tau$. As an application, we prove a weak version of the Cappell-Miller conjecture. 


\section{The Quadratic Form on the Determinant Line of a Finite-Dimensional Complex}

In this section, we define a canonical quadratic form on a finite-dimensional complex with involution.

\subsection{The construction of a quadratic form}

Let

$$
\left(C^{\bullet}, \partial\right): 0 \rightarrow C^{0} \stackrel{\partial}{\longrightarrow} C^{1} \stackrel{\partial}{\longrightarrow} \cdots \stackrel{\partial}{\longrightarrow} C^{d} \rightarrow 0
$$

be a complex of finite-dimensional complex vector spaces of odd length $d=2 r-1$. A chirality operator $\Gamma: C^{\bullet} \rightarrow C^{\bullet}$ is an involution such that $\Gamma\left(C^{j}\right)=C^{d-j}$, for all $j=0, \ldots, d$. Consider the determinant line

$$
\operatorname{Det}\left(C^{\bullet}\right):=\bigotimes_{j=0}^{d} \operatorname{Det}\left(C^{j}\right)^{(-1)^{j}}
$$

where $\operatorname{Det}\left(C^{j}\right)^{-1}:=\operatorname{Hom}\left(\operatorname{Det}\left(C^{j}\right), \mathbb{C}\right)$ denotes the dual of $C^{j}$. For an element $c_{j} \in \operatorname{Det}\left(C^{j}\right)$ we denote by $c_{j}^{-1}$ the unique element in $\operatorname{Det}\left(C^{j}\right)^{-1}$ satisfying $C_{j}^{-1}\left(c_{j}\right)=1$. We also denote by $\Gamma c_{j} \in \operatorname{Det}\left(C^{d-j}\right)$ the image of $c_{j}$ under the map $\operatorname{Det}\left(C^{j}\right) \rightarrow \operatorname{Det}\left(C^{d-j}\right)$ induced by $\Gamma: C^{j} \rightarrow$ $C^{d-j}$.

Denote by $H^{\bullet}(\partial)$ the cohomology of the complex $\left(C^{\bullet}, \partial\right)$. Let

$$
\phi_{C} \cdot: \operatorname{Det}\left(C^{\bullet}\right) \longrightarrow \operatorname{Det}\left(H^{\bullet}(\partial)\right)
$$

be the canonical isomorphism, cf. [22]. (In [7] we used a sign refined version of this isomorphism, but we don't need this more complicated version in the present paper.)

Note that any element $c \in \operatorname{Det}\left(C^{\bullet}\right)$ can be written in a form $c=c_{0} \otimes c_{1}^{-1} \otimes \cdots \otimes c_{d}^{-1}$, where $c_{j} \in \operatorname{Det}\left(C^{j}\right)$. Hence, any element of $\operatorname{Det}\left(H^{\bullet}(\partial)\right)$ can be written as $\phi_{C} \cdot\left(c_{0} \otimes C_{1}^{-1} \otimes \cdots\right.$ $\left.\otimes c_{d}^{-1}\right)$.

Definition 2.2. The torsion quadratic form $\tau_{\Gamma}$ of the pair $\left(C^{\bullet}, \Gamma\right)$ is the unique quadratic form on $\operatorname{Det}\left(H^{\bullet}(\partial)\right)$ such that

$$
\tau_{\Gamma}\left(\phi_{C} \cdot\left(c_{0} \otimes c_{1}^{-1} \otimes \cdots \otimes c_{d}^{-1}\right)\right)=\prod_{j=0}^{d}\left[c_{j}^{-1}\left(\Gamma c_{d-j}\right)\right]^{(-1)^{j+1}}
$$




\subsection{Relationship with the refined torsion}

In [7] we introduced a canonical element of $\operatorname{Det}\left(H^{\bullet}(\partial)\right)$, called the refined torsion of the pair $\left(C^{\bullet}, \Gamma\right)$, as follows. For each $j=0, \ldots, r-1$, fix an element $c_{j} \in \operatorname{Det}\left(C^{j}\right)$ and set

$$
c_{\Gamma}:=(-1)^{\mathcal{R}\left(C^{\bullet}\right)} \cdot c_{0} \otimes c_{1}^{-1} \otimes \cdots \otimes c_{r-1}^{(-1)^{r-1}} \otimes\left(\Gamma c_{r-1}\right)^{(-1)^{r}} \otimes\left(\Gamma c_{r-2}\right)^{(-1)^{r-1}} \otimes \cdots \otimes\left(\Gamma c_{0}\right)^{-1}
$$

of $\operatorname{Det}\left(C^{\bullet}\right)$, where

$$
\mathcal{R}\left(C^{\bullet}\right)=\frac{1}{2} \sum_{j=0}^{r-1} \operatorname{dim} C^{j} \cdot\left(\operatorname{dim} C^{j}+(-1)^{r+j}\right) .
$$

It is easy to see that $c_{\Gamma}$ is independent of the choice of $c_{0}, \ldots, c_{r-1}$. The refined torsion of the pair $\left(C^{\bullet}, \Gamma\right)$ is the element

$$
\rho_{\Gamma}=\rho_{C^{\bullet}, \Gamma}:=\phi_{C} \cdot\left(C_{\Gamma}\right) \in \operatorname{Det}\left(H^{\bullet}(\partial)\right) .
$$

It follows immediately from (2.3) and (2.6) that

$$
\tau_{\Gamma}\left(\rho_{\Gamma}\right)=1
$$

\subsection{An acyclic complex}

Suppose the complex $\left(C^{\bullet}, \partial\right)$ is acyclic. Then $\operatorname{Det}\left(H^{\bullet}(\partial)\right)$ is naturally isomorphic to $\mathbb{C}$. Using this isomorphism, we identify $\tau_{\Gamma}$ with the complex number

$$
\hat{\tau}_{\Gamma}:=\tau_{\Gamma}(1) \in \mathbb{C} \backslash\{0\}, \quad 1 \in \mathbb{C} \simeq \operatorname{Det}\left(H^{\bullet}(\partial)\right) .
$$

\subsection{Calculation of the refined torsion of a finite-dimensional complex}

To compute the refined torsion, we introduce the operator

$$
\mathcal{B}:=\Gamma \partial+\partial \Gamma
$$

This operator is a finite-dimensional analogue of the signature operator on an odddimensional manifold; see [1, p. 44], [2, p. 405], [20, pp. 64-65], and Section 3 of this 
paper. Then

$$
\mathcal{B}^{2}=\Gamma \partial \Gamma \partial+\partial \Gamma \partial \Gamma
$$

Remark 2.6. In many interesting applications, cf. Section 3, there exists a scalar product on $C^{\bullet}$ such that the adjoint of $\partial$ satisfies $\partial^{*}=\Gamma \partial \Gamma$. Then $\mathcal{B}^{2}$ is equal to the Laplacian of the complex $C^{\bullet}$.

Let us first treat the case where the signature operator $\mathcal{B}$ is bijective.

Lemma 2.7. Suppose that the operator $\mathcal{B}$ is invertible. Then the complex $\left(C^{\bullet}, \partial\right)$ is acyclic and the complex number $\hat{\tau}_{\Gamma}$, cf. (2.8), is given by

$$
\hat{\tau}_{\Gamma}=\prod_{j=0}^{d} \operatorname{Det}\left(\left.\mathcal{B}^{2}\right|_{C^{j}}\right)^{(-1)^{j} j}
$$

Proof. Since $\Gamma^{2}=\mathrm{Id}$, for every $a \in \operatorname{Det}\left(C^{\bullet}\right), b \in \operatorname{Det}\left(C^{d-\bullet}\right)$, we have

$$
a^{-1}(\Gamma b)=(\Gamma a)^{-1}(b)=\frac{1}{b^{-1}(\Gamma a)} .
$$

Hence, for all $j=0, \ldots, d$,

$$
\left[c_{j}^{-1}\left(\Gamma c_{d-j}\right)\right]^{(-1)^{j+1}}=\left[c_{d-j}^{-1}\left(\Gamma c_{j}\right)\right]^{(-1)^{d-j+1}}
$$

and the definition (2.3) of $\tau_{\Gamma}$ can be rewritten as

$$
\tau_{\Gamma}\left(\phi_{C} \cdot\left(c_{0} \otimes c_{1}^{-1} \otimes \cdots \otimes c_{d}^{-1}\right)\right)=\left[\prod_{j=0}^{r-1}\left[c_{j}^{-1}\left(\Gamma c_{d-j}\right)\right]^{(-1)^{j+1}}\right]^{2}
$$

As, by assumption, the operator $\mathcal{B}=\Gamma \partial+\partial \Gamma$ is invertible, for each $j=0, \ldots, n$ we have a direct sum decomposition

$$
C^{j}=A^{j} \oplus B^{j}
$$

where $A^{j}=\operatorname{Ker}\left(\left.\partial\right|_{C^{j}}\right)$ and $B^{j}=\Gamma \partial\left(C^{d-j-1}\right)$. It follows that the complex $\left(C^{\bullet}, \partial\right)$ is acyclic and $A^{j}=\partial\left(B^{j-1}\right)$ for all $j=1, \ldots, d$. Set $n_{j}=\operatorname{dim} B^{j}$. Then $n_{j}=n_{d-j-1}$ and $\operatorname{dim} A^{j}=n_{j-1}$. 
For $j=0, \ldots, r-1$ choose a basis $\left\{b_{1}^{j}, \ldots, b_{n_{j}}^{j}\right\}$ of $B^{j}$. For $j=r, \ldots, d-1$ set $b_{i}^{j}=\Gamma \partial b_{i}^{d-j-1}$. Then for any $j=0, \ldots, d-1,\left\{b_{1}^{j}, \ldots, b_{n_{j}}^{j}\right\}$ is a basis of $B^{j}$. It follows that $\left\{\partial b_{1}^{j-1}, \ldots, \partial b_{n_{j-1}}^{j-1}\right\}$ is a basis of $A^{j}$, for $j=1, \ldots, d$. Hence,

$$
\left\{\partial b_{1}^{j-1}, \ldots, \partial b_{n_{j-1}}^{j-1}, b_{1}^{j}, \ldots, b_{n_{j}}^{j}\right\}
$$

is a basis of $C^{j}(j=1, \ldots, d-1),\left\{b_{1}^{0}, \ldots, b_{n_{0}}^{0}\right\}$ is the basis of $C^{0}$, and $\left\{\partial b_{1}^{d-1}, \ldots, \partial b_{n_{d-1}}^{d-1}\right\}$ is the basis of $C^{d}$. Set

$$
c_{0}=b_{1}^{0} \wedge \cdots \wedge b_{n_{0}}^{0}, \quad c_{d}=\partial b_{1}^{d-1} \wedge \cdots \wedge \partial b_{n_{d-1}}^{d-1},
$$

and, for $j=1, \ldots, d-1$,

$$
c_{j}=\partial b_{1}^{j-1} \wedge \cdots \wedge \partial b_{n_{j-1}}^{j-1} \wedge b_{1}^{j} \wedge \cdots \wedge b_{n_{j}}^{j} \in \operatorname{Det}\left(C^{j}\right) .
$$

By the definition of the map $\phi_{C^{\bullet}}: \operatorname{Det}\left(C^{\bullet}\right) \rightarrow \operatorname{Det}\left(H^{\bullet}(\partial)\right) \simeq \mathbb{C}$

$$
\phi_{C} \cdot\left(c_{0} \otimes c_{1}^{-1} \otimes \cdots \otimes c_{d}^{-1}\right)=1 \in \mathbb{C}
$$

Therefore, by (2.8) and (2.12),

$$
\hat{\tau}_{\Gamma}=\left[\prod_{j=0}^{r-1}\left[c_{j}^{-1}\left(\Gamma c_{d-j}\right)\right]^{(-1)^{j+1}}\right]^{2} .
$$

We now need to compute the numbers $c_{j}^{-1}\left(\Gamma c_{d-j}\right)$. Assume first, that $j=1, \ldots, r-$ 2. Then $c_{j}^{-1}\left(\Gamma c_{d-j}\right)$ is equal to the determinant of the operator $S_{j}: C^{j} \rightarrow C^{j}$, which transforms the basis $\left\{\partial b_{1}^{j-1}, \ldots, \partial b_{n_{j-1}}^{j-1}, b_{1}^{j}, \ldots, b_{n_{j}}^{j}\right\}$ to the basis

$$
\left\{\Gamma \partial b_{1}^{d-j-1}, \ldots, \Gamma \partial b_{n_{d-j-1}}^{d-j-1}, \Gamma b_{1}^{d-j}, \ldots, \Gamma b_{n_{d-j}}^{d-j}\right\}=\left\{\Gamma \partial \Gamma \partial b_{1}^{j}, \ldots, \Gamma \partial \Gamma \partial b_{n_{j^{\prime}}}^{j} \partial b_{1}^{j-1}, \ldots, \partial b_{n_{j-1}}^{j-1}\right\} .
$$

Here we used that, by construction, $\Gamma \partial b_{i}^{j}=b_{i}^{d-j-1}$, for any $i=1, \ldots, n_{j}$ and $b_{i}^{d-j}=\Gamma \partial b_{i}^{j-1}$ for any $i=1, \ldots, n_{j-1}$. We conclude that

$$
c_{j}^{-1}\left(\Gamma c_{d-j}\right)=\operatorname{Det}\left(S_{j}\right)= \pm \operatorname{Det}\left(\left.\Gamma \partial \Gamma \partial\right|_{B^{j}}\right), \quad j=1, \ldots, r-2 .
$$


Similarly, $c_{0}^{-1}\left(\Gamma c_{d}\right)$ is the determinant of the operator, which transforms the basis $\left\{b_{1}^{0}, \ldots, b_{n_{0}}^{0}\right\}$ to the basis

$$
\left\{\Gamma \partial b_{1}^{d-1}, \ldots, \Gamma \partial b_{n_{d-1}}^{d-1}\right\}=\left\{\Gamma \partial \Gamma \partial b_{1}^{0}, \ldots, \Gamma \partial \Gamma \partial b_{n_{0}}^{0}\right\}
$$

Thus,

$$
c_{0}^{-1}\left(\Gamma c_{d}\right)=\operatorname{Det}\left(\left.\Gamma \partial \Gamma \partial\right|_{B^{0}}\right)
$$

Finally, $c_{r-1}^{-1}\left(\Gamma c_{r}\right)$ is equal to the determinant of the operator, which transforms the basis $\left\{\partial b_{1}^{r-2}, \ldots, \partial b_{n_{r-2}}^{r-2}, b_{1}^{r-1}, \ldots, b_{n_{r-1}}^{r-1}\right\}$ to the basis

$$
\left\{\Gamma \partial b_{1}^{r-1}, \ldots, \Gamma \partial b_{n_{r-1}}^{r-1}, \Gamma b_{1}^{r}, \ldots, \Gamma b_{n_{r}}^{r}\right\}=\left\{\Gamma \partial b_{1}^{r-1}, \ldots, \Gamma \partial b_{n_{r-1}}^{r-1}, \partial b_{1}^{r-2}, \ldots, \partial b_{n_{r-2}}^{r-2}\right\}
$$

and, hence, is equal to $\pm \operatorname{Det}\left(\left.\Gamma \partial\right|_{B^{r-1}}\right)$. Therefore,

$$
\left[c_{r-1}^{-1}\left(\Gamma c_{r}\right)\right]^{2}=\operatorname{Det}\left(\left.\Gamma \partial\right|_{B^{r-1}}\right)^{2}=\operatorname{Det}\left(\left.\Gamma \partial \Gamma \partial\right|_{B^{r-1}}\right)
$$

Combining Equations (2.13)-(2.16), we obtain

$$
\hat{\tau}_{\Gamma}=\left[\prod_{j=0}^{r-2}\left[\operatorname{Det}\left(\left.\Gamma \partial \Gamma \partial\right|_{B^{j}}\right)\right]^{(-1)^{j+1}}\right]^{2} \cdot \operatorname{Det}\left(\left.\Gamma \partial \Gamma \partial\right|_{B^{r-1}}\right) .
$$

The isomorphism $Г \partial: B^{j} \rightarrow B^{d-j-1}$ intertwines the operators $\left.Г \partial Г \partial\right|_{B^{j}}$ and $\left.\Gamma \partial \Gamma \partial\right|_{B^{d-j-1}}$. Hence,

$$
\operatorname{Det}\left(\left.\Gamma \partial \Gamma \partial\right|_{B^{j}}\right)=\operatorname{Det}\left(\left.\Gamma \partial \Gamma \partial\right|_{B^{d-j-1}}\right)
$$

and (2.17) can be rewritten as

$$
\hat{\tau}_{\Gamma}=\prod_{j=0}^{d-1}\left[\operatorname{Det}\left(\left.\Gamma \partial \Gamma \partial\right|_{B^{j}}\right)\right]^{(-1)^{j+1}}
$$


The isomorphism $\partial: B^{j-1} \rightarrow A^{j}$ intertwines the operators $\left.\Gamma \partial \Gamma \partial\right|_{B^{j-1}}$ and $\left.\partial \Gamma \partial \Gamma\right|_{A^{j}}$. Hence,

$$
\operatorname{Det}\left(\left.\Gamma \partial \Gamma \partial\right|_{B^{j-1}}\right)=\operatorname{Det}\left(\left.\partial \Gamma \partial \Gamma\right|_{A^{j}}\right), \quad j=1, \ldots, d .
$$

Thus, from (2.10), we conclude that

$$
\operatorname{Det}\left(\left.\mathcal{B}^{2}\right|_{C^{0}}\right)=\operatorname{Det}\left(\left.\Gamma \partial \Gamma \partial\right|_{B^{0}}\right), \quad \operatorname{Det}\left(\left.\mathcal{B}^{2}\right|_{C^{d}}\right)=\operatorname{Det}\left(\left.\Gamma \partial \Gamma \partial\right|_{B^{d-1}}\right),
$$

and, for $j=1, \ldots, d-1$,

$$
\operatorname{Det}\left(\left.\mathcal{B}^{2}\right|_{C^{j}}\right)=\operatorname{Det}\left(\left.\Gamma \partial \Gamma \partial\right|_{B^{j}}\right) \cdot \operatorname{Det}\left(\left.\partial \Gamma \partial \Gamma\right|_{A^{j}}\right)=\operatorname{Det}\left(\left.\Gamma \partial \Gamma \partial\right|_{B^{j}}\right) \cdot \operatorname{Det}\left(\left.\Gamma \partial \Gamma \partial\right|_{B^{j-1}}\right) .
$$

Therefore,

$$
\begin{aligned}
\prod_{j=0}^{d} \operatorname{Det}\left(\left.\mathcal{B}^{2}\right|_{C^{j}}\right)^{(-1)^{j} j} & =\prod_{j=0}^{d-1} \operatorname{Det}\left(\operatorname{Det}\left(\left.\Gamma \partial \Gamma \partial\right|_{B^{j}}\right)\right)^{(-1)^{j} j} \cdot \prod_{j=1}^{d} \operatorname{Det}\left(\operatorname{Det}\left(\left.\Gamma \partial \Gamma \partial\right|_{B^{j-1}}\right)\right)^{(-1)^{j} j} \\
& =\prod_{j=0}^{d-1} \operatorname{Det}\left(\operatorname{Det}\left(\left.\Gamma \partial \Gamma \partial\right|_{B^{j}}\right)\right)^{(-1)^{j+1}} \cdot
\end{aligned}
$$

Combining (2.19) and (2.18), we obtain (2.11).

To compute the torsion quadratic form in the case $\mathcal{B}$ is not bijective, note that, for $j=0, \ldots, d$, the operator $\mathcal{B}^{2}$ maps $C^{j}$ into itself. For each $j=0, \ldots, d$ and an arbitrary interval $\mathcal{J}$, denote by $C_{\mathfrak{J}}^{j} \subset C^{j}$ the linear span of the generalized eigenvectors of the restriction of $\mathcal{B}^{2}$ to $C^{j}$, corresponding to eigenvalues $\lambda$ with $|\lambda| \in \mathcal{J}$. Since both operators, $\Gamma$ and $\partial$, commute with $\mathcal{B}$ (and, hence, with $\left.\mathcal{B}^{2}\right), \Gamma\left(C_{\mathcal{J}}^{j}\right) \subset C_{\mathcal{J}}^{d-j}$ and $\partial\left(C_{\mathcal{J}}^{j}\right) \subset C_{\mathcal{J}}^{j+1}$. Hence, we obtain a subcomplex $C_{\mathcal{J}}^{\bullet}$ of $C^{\bullet}$ and the restriction $\Gamma_{\mathcal{J}}$ of $\Gamma$ to $C_{\mathfrak{J}}^{\bullet}$ is a chirality operator for $C_{\mathcal{J}}$. We denote by $H_{\mathcal{J}}(\partial)$ the cohomology of the complex $\left(C_{\mathfrak{J}}, \partial_{\mathcal{J}}\right)$.

Denote by $\partial_{\mathcal{J}}$ and $\mathcal{B}_{\mathcal{J}}$ the restrictions of $\partial$ and $\mathcal{B}$ to $C_{\mathfrak{J}}^{\bullet}$. Then $B_{\mathcal{J}}=\Gamma_{\mathcal{J}} \partial_{\mathcal{J}}+\partial_{\mathcal{J}} \Gamma_{\mathcal{J}}$ and one easily shows (cf. Lemma 5.8 of [7]) that $\left(C_{\mathcal{J}}^{\bullet}, \partial_{\mathcal{J}}\right)$ is acyclic if $0 \notin \mathcal{J}$.

For each $\lambda \geq 0, C^{\bullet}=C_{[0, \lambda]}^{\bullet} \oplus C_{(\lambda, \infty)}^{\bullet}$ and $H_{(\lambda, \infty)}^{\bullet}(\partial)=0$ whereas $H_{[0, \lambda]}^{\bullet}(\partial) \simeq H^{\bullet}(\partial)$. Hence, there are canonical isomorphisms

$$
\Phi_{\lambda}: \operatorname{Det}\left(H_{(\lambda, \infty)}^{\bullet}(\partial)\right) \longrightarrow \mathbb{C}, \quad \Psi_{\lambda}: \operatorname{Det}\left(H_{[0, \lambda]}^{\bullet}(\partial)\right) \longrightarrow \operatorname{Det}\left(H^{\bullet}(\partial)\right)
$$


In the sequel, we will write $t$ for $\Phi_{\lambda}(t) \in \mathbb{C}$.

Lemma 2.8. For every $x \in \operatorname{Det}\left(H^{\bullet}(\partial)\right)$ and every $\lambda \geq 0$, we have

$$
\tau_{\Gamma}(x)=\left[\prod_{j=0}^{d} \operatorname{Det}\left(\left.\mathcal{B}_{(\lambda, \infty)}^{2}\right|_{C_{(\lambda, \infty)}^{j}}\right)^{(-1)^{j} j}\right] \cdot \tau_{\Gamma_{[0, \lambda]}}\left(\Psi_{\lambda}^{-1}(x)\right) .
$$

In particular, the right-hand side of (2.20) is independent of $\lambda \geq 0$.

Proof. For each $j=0, \ldots, d$ fix $c_{j}^{\prime} \in \operatorname{Det}\left(C_{[0, \lambda]}^{j}\right)$ and $c_{j}^{\prime \prime} \in \operatorname{Det}\left(C_{(\lambda, \infty)}^{j}\right)$. Then, using the natural isomorphism

$$
\operatorname{Det}\left(C_{[0, \lambda]}^{j}\right) \otimes \operatorname{Det}\left(C_{(\lambda, \infty)}^{j}\right) \simeq \operatorname{Det}\left(C_{[0, \lambda]}^{j} \oplus C_{(\lambda, \infty)}^{j}\right)=\operatorname{Det}\left(C^{j}\right),
$$

we can regard the tensor product $c_{j}:=c_{j}^{\prime} \otimes c_{j}^{\prime \prime}$ as an element of $\operatorname{Det}\left(C^{j}\right)$. Applying (2.3) twice, we obtain

$$
\begin{aligned}
\tau_{\Gamma}\left(\phi_{C} \cdot\left(c_{0} \otimes c_{1}^{-1} \otimes \cdots \otimes c_{d}^{-1}\right)\right)=\prod_{j=0}^{d}\left[c_{j}^{-1}\left(\Gamma c_{d-j}\right)\right]^{(-1)^{j+1}} \\
=\prod_{j=0}^{d}\left[\left(c_{j}^{\prime}\right)^{-1}\left(\Gamma c_{d-j}^{\prime}\right)\right]^{(-1)^{j+1}} \cdot \prod_{j=0}^{d}\left[\left(c_{j}^{\prime \prime}\right)^{-1}\left(\Gamma c_{d-j}^{\prime \prime}\right)\right]^{(-1)^{j+1}} \\
=\tau_{\Gamma_{[0, \lambda]}}\left(\phi_{C_{[0, \lambda]}^{\cdot}}\left(c_{0}^{\prime} \otimes\left(c_{1}^{\prime}\right)^{-1} \otimes \cdots \otimes\left(c_{d}^{\prime}\right)^{-1}\right)\right) \cdot \tau_{\Gamma_{(\lambda, \infty)}}\left(\left(\phi_{C_{(\lambda, \infty)}}\left(c_{0}^{\prime \prime} \otimes\left(c_{1}^{\prime \prime}\right)^{-1} \otimes \cdots \otimes\left(c_{d}^{\prime \prime}\right)^{-1}\right)\right) .\right.
\end{aligned}
$$

Let us now choose $c_{j}^{\prime}$ and $c_{j}^{\prime \prime}(j=0, \ldots, d)$ such that $\phi_{C}^{\bullet}\left(c_{0} \otimes c_{1}^{-1} \otimes \cdots \otimes c_{d}^{-1}\right)=x$ and

$$
\Phi_{\lambda} \circ \phi_{C_{(\lambda, \infty)}}\left(c_{0}^{\prime \prime} \otimes\left(c_{1}^{\prime \prime}\right)^{-1} \otimes \cdots \otimes\left(c_{d}^{\prime \prime}\right)^{-1}\right)=1 .
$$

Then

$$
\Psi_{\lambda} \circ \phi_{C_{[0, \lambda]}^{\bullet}}\left(c_{0}^{\prime} \otimes\left(c_{1}^{\prime}\right)^{-1} \otimes \cdots \otimes\left(c_{d}^{\prime}\right)^{-1}\right)= \pm X
$$

and from (2.11) we get

$$
\tau_{\Gamma_{(\lambda, \infty)}} \circ \phi_{C_{(\lambda, \infty)}}\left(c_{0}^{\prime \prime} \otimes\left(c_{1}^{\prime \prime}\right)^{-1} \otimes \cdots \otimes\left(c_{d}^{\prime \prime}\right)^{-1}\right)=\prod_{j=0}^{d} \operatorname{Det}\left(\left.\mathcal{B}_{(\lambda, \infty)}^{2}\right|_{C_{(\lambda, \infty)}^{j}}\right)^{(-1)^{j} j} .
$$

Hence, (2.20) is equivalent to (2.21). 


\section{The Quadratic Form Associated with the Square of the Odd Signature Operator}

Let $E \rightarrow M$ be a complex vector bundle over a closed oriented manifold of odd dimension $d=2 r-1$ and let $\nabla$ be a flat connection on $E$. Further, let $\Omega^{\bullet}(M, E)$ denote the de Rham complex of $E$-valued differential forms on $M$. For a given Riemannian metric $g^{M}$ on $M$, denote by

$$
\Gamma=\Gamma\left(g^{M}\right): \Omega^{\bullet}(M, E) \longrightarrow \Omega^{\bullet}(M, E)
$$

the chirality operator (cf. [3, Section 3]), defined in terms of the Hodge $*$-operator by the formula

$$
\Gamma \omega:=i^{r}(-1)^{\frac{k(k+1)}{2}} * \omega, \quad \omega \in \Omega^{k}(M, E) .
$$

The odd signature operator introduced by Atiyah, Patodi, and Singer [1, 2] (see also [20]) is the first-order elliptic differential operator $\mathcal{B}: \Omega^{\bullet}(M, E) \rightarrow \Omega^{\bullet}(M, E)$, given by

$$
\mathcal{B}=\mathcal{B}\left(\nabla, g^{M}\right) \stackrel{\text { Def }}{=} \Gamma \nabla+\nabla \Gamma .
$$

Note that the operator $\mathcal{B}$ is elliptic and its leading symbol is self-adjoint with respect to any Hermitian metric on $E$. Remark also that $\mathcal{B}^{2}$ maps $\Omega^{j}(M, E)$ into itself for every $j=0, \ldots, d$. We denote by $\left(\mathcal{B}^{2}\right)_{j}$ the restriction of $\mathcal{B}^{2}$ to $\Omega^{j}(M, E)$.

For an interval $\mathcal{J} \subset[0, \infty)$, we denote by $\Omega_{\mathcal{J}}^{j}(M, E)$ the image of $\Omega^{j}(M, E)$ under the spectral projection of $\left(\mathcal{B}^{2}\right)_{j}$ corresponding to the eigenvalues whose absolute value lie in $\mathcal{J}$. The space $\Omega_{\mathcal{J}}^{j}(M, E)$ contains the span of the generalized eigenforms of $\left(\mathcal{B}^{2}\right)_{j}$ corresponding to eigenvalues whose absolute value lies in $\mathcal{J}$ and coincides with this span if the interval $\mathcal{J}$ is bounded. In particular, since $\mathcal{B}$ is elliptic, if $\mathcal{J}$ is bounded, then the dimension of $\Omega_{\mathfrak{J}}^{j}(M, E)$ is finite. Since $\mathcal{B}^{2}$ and $\nabla$ commute, $\Omega_{\mathfrak{j}}^{\bullet}(M, E)$ is a subcomplex of the de Rham complex $\Omega^{\bullet}(M, E)$.

For each $\lambda \geq 0$, we have

$$
\Omega^{\bullet}(M, E)=\Omega_{[0, \lambda]}^{\bullet}(M, E) \oplus \Omega_{(\lambda, \infty)}^{\bullet}(M, E) .
$$

The complex $\Omega_{(\lambda, \infty)}^{\bullet}(M, E)$ is clearly acyclic. Hence, the cohomology $H_{[0, \lambda]}^{\bullet}(M, E)$ of the complex $\Omega_{[0, \lambda]}^{\bullet}(M, E)$ is naturally isomorphic to the cohomology $H^{\bullet}(M, E)$ of $\Omega^{\bullet}(M, E)$. 
Further, as $\Gamma$ commutes with $\mathcal{B}^{2}$, it preserves the space $\Omega_{[0, \lambda]}(M, E)$ and the restriction $\Gamma_{[0, \lambda]}$ of $\Gamma$ to this space is a chirality operator on $\Omega_{[0, \lambda]}^{\bullet}(M, E)$.

Denote by $\mathcal{B}_{\mathcal{J}, j}^{2}$ the restrictions of $\mathcal{B}^{2}$ to $\Omega_{\mathcal{J}}^{j}(M, E)$. Let $\theta \in(0,2 \pi)$ be an Agmon angle for $\mathcal{B}_{\mathcal{J}}^{2}$, cf. [25], and denote by $\operatorname{Det}_{\theta}\left(\mathcal{B}_{(\lambda, \infty), j}^{2}\right)$ the $\zeta$-regularized determinant of the operator $\mathcal{B}_{(\lambda, \infty), j}^{2}$ defined using the Agmon angle $\theta$. Since the leading symbol of $\mathcal{B}_{(\lambda, \infty), j}^{2}$ is positive definite this determinant is independent of the choice of $\theta$.

For any $0 \leq \lambda \leq \mu<\infty$, one easily sees that

$$
\prod_{j=0}^{d} \operatorname{Det}_{\theta}\left(\mathcal{B}_{(\lambda, \infty), j}^{2}\right)^{(-1)^{j} j}=\left[\prod_{j=0}^{d} \operatorname{Det}_{\theta}\left(\mathcal{B}_{(\lambda, \mu], j}^{2}\right)^{(-1)^{j} j}\right] \cdot\left[\prod_{j=0}^{d} \operatorname{Det}_{\theta}\left(\mathcal{B}_{(\mu, \infty), j}^{2}\right)^{(-1)^{j} j}\right] .
$$

For any given $\lambda \geq 0$, denote by $\tau_{\Gamma_{[0, \lambda]}}$ the quadratic form on the determinant line of $H_{[0, \lambda]}^{\bullet}(M, E)$ associated with the chirality operator $\Gamma_{[0, \lambda]}$, cf. Definition 2.2. In view of (2.20) and (3.2), the product

$$
\tau=\tau(\nabla):=\left[\prod_{j=0}^{d} \operatorname{Det}\left(\mathcal{B}_{(\lambda, \infty), j}^{2}\right)^{(-1)^{j} j}\right] \cdot \tau_{\Gamma_{[0, \lambda]}}
$$

viewed as a quadratic form on $\operatorname{Det}\left(H^{\bullet}(M, E)\right)$ is independent of the choice of $\lambda \geq 0$. It is also independent of the choice of the Agmon angle $\theta \in(0,2 \pi)$ of $\mathcal{B}_{(\lambda, \infty)}^{2}$.

Definition 3.1. The quadratic form (3.3) on the determinant line of $H^{\bullet}(M, E)$ is called the torsion quadratic form.

Theorem 3.1. The torsion quadratic form $\tau$ is independent of the Riemannian metric $g^{M}$.

Proof. Suppose that $g_{t}^{M}, t \in \mathbb{R}$, is a smooth family of Riemannian metrics on $M$ and let $\tau_{t}$ denote the torsion quadratic form corresponding to the metric $g_{t}^{M}$. We need to show that $\tau_{t}$ is independent of $t$.

Let $\Gamma_{t}$ denote the chirality operator corresponding to the metric $g_{t}^{M}$, cf. (3.1), and let $\mathcal{B}(t)=\mathcal{B}\left(\nabla, g_{t}^{M}\right)$ denote the odd signature operator corresponding to $\Gamma_{t}$.

Fix $t_{0} \in \mathbb{R}$ and choose $\lambda \geq 0$ so that there are no eigenvalues of $\mathcal{B}\left(t_{0}\right)^{2}$ whose absolute values are equal to $\lambda$. Then there exists $\delta>0$ such that the same is true for all $t \in\left(t_{0}-\delta, t_{0}+\delta\right)$. In particular, if we denote by $\Omega_{[0, \lambda], t}^{\bullet}(M, E)$ the span of the 
generalized eigenvectors of $\mathcal{B}(t)^{2}$ corresponding to eigenvalues with absolute value $\leq \lambda$, then $\operatorname{dim} \Omega_{[0, \lambda], t}^{\bullet}(M, E)$ is independent of $t \in\left(t_{0}-\delta, t_{0}+\delta\right)$.

Let $\rho_{\Gamma_{t,[0, \lambda]}}$ denote the refined torsion of the pair $\left(\Omega_{[0, \lambda], t}^{0}(M, E), \Gamma_{t}\right)$, cf. Subsection 2.2. As above, we shall view $\rho_{\Gamma_{t, 0, \lambda]}}$ as an element of $\operatorname{Det}\left(H^{\bullet}(M, E)\right)$ via the canonical isomorphism between $H^{\bullet}(M, E)$ and $H_{[0, \lambda]}^{\bullet}(M, E)$.

In [7] we fixed a particular square root of $\prod_{j=0}^{d} \operatorname{Det}_{\theta}\left(\mathcal{B}(t)_{(\lambda, \infty), j}^{2}\right)^{(-1)^{j+1} j}$ (in [7] it is denoted by $\left.\mathrm{e}^{\xi_{\lambda}\left(t, \theta_{0}\right)}\right)$. By Lemma 9.2 of [7], the element

$$
\rho:=\sqrt{\prod_{j=0}^{d} \operatorname{Det}_{\theta}\left(\mathcal{B}(t)_{(\lambda, \infty), j}^{2}\right)^{(-1)^{j+1} j}} \cdot \rho_{\Gamma_{t,[0, \lambda]}} \in \operatorname{Det}\left(H^{\bullet}(M, E)\right)
$$

is independent of $t \in\left(t_{0}-\delta, t_{0}+\delta\right)$.

Let $\tau_{\Gamma_{t, 10, \lambda]}}$ denote the torsion quadratic form of the pair $\left(\Omega_{[0, \lambda], t}^{\bullet}(M, E), \Gamma_{t}\right)$. By $(2.7)$ we have

$$
\tau_{t}(\rho)=\prod_{j=0}^{d} \operatorname{Det}\left(\mathcal{B}(t)_{[\lambda, \infty), j}^{2}\right)^{(-1)^{j} j} \cdot \tau_{\Gamma_{t,[0, \lambda]}}(\rho)=\tau_{\tau_{t,[0, \lambda]}}\left(\rho_{\Gamma_{t,[0, \lambda]}}\right)=1,
$$

where, in the latter equality, we used (2.7). Thus $\tau_{t}(\rho)$ is independent of $t \in\left(t_{0}-\delta, t_{0}+\delta\right)$. Since this is true for an arbitrary value of $t_{0}$, the theorem is proven.

Remark 3.2. One can easily give a direct proof of Theorem 3.1, avoiding any references to [7]. One only needs to repeat most of the computations of the proof of Lemma 9.2 of [7]. However, to save space we preferred to use this lemma, rather than repeat its proof.

\section{The Relationship with Burghelea-Haller and Farber-Turaev Torsions}

In this section, we show that the torsion quadratic form $\tau$ coincides with the quadratic form defined in [5] and use the results of [5] to establish the relationship between $\tau$ and the Burghelea-Haller and Farber-Turaev torsions.

\subsection{Relationship with the refined analytic torsion}

Let $\eta(\nabla)=\eta\left(\nabla, g^{M}\right)$ denote the $\eta$-invariant of the restriction of the odd signature operator $\mathcal{B}=\mathcal{B}\left(\nabla, g^{M}\right)$ to the even forms, see [20], [8, Section 4], [7, Section 6.15], or [5, Section 2.9] 
for the definition of the $\eta$-invariant of a non-self-adjoint operator. Let $\eta_{\text {trivial }}$ be the $\eta$ invariant of trivial line bundle over $M$. Let $\rho_{\text {an }}=\rho_{\text {an }}(\nabla) \in \operatorname{Det}\left(H^{\bullet}(M, E)\right)$ denote the refined analytic torsion of $(E, \nabla)$, cf. [7, Definition 9.8].

Proposition 4.2. $\tau_{\nabla}\left(\rho_{\mathrm{an}}(\nabla)\right)=e^{-2 \pi i\left(\eta(\nabla)-\operatorname{rank} E \cdot \eta_{\text {trivial }}\right)}$.

It follows that the torsion quadratic form $\tau$ coincides with the quadratic form defined by Equation (1.1) of [5].

Proof. Recall that the element $\rho \in \operatorname{Det}\left(H^{\bullet}(M, E)\right)$ is defined in (3.4). From definition of the refined analytic torsion, [7, Definition 9.8], and formulae (9-5) and (10-21) of [7], we conclude that

$$
\rho_{\mathrm{an}}(\nabla)= \pm \rho \cdot e^{-\pi i\left(\eta(\nabla)-\operatorname{rank} E \cdot \eta_{\text {trivial }}\right)}
$$

Hence, the statement of the proposition follows immediately from (3.5).

\subsection{Relationship with the Burghelea-Haller torsion}

Burghelea and Haller [11, 12] have introduced a refinement of the square of the RaySinger torsion for a closed manifold of arbitrary dimension, provided that the complex vector bundle $E$ admits a nondegenerate complex-valued symmetric bilinear form $b$. They defined a complex-valued quadratic form

$$
\tau^{\mathrm{BH}}=\tau_{b, \nabla}^{\mathrm{BH}}
$$

on the determinant line $\operatorname{Det}\left(H^{\bullet}(M, E)\right)$, which depends holomorphically on the flat connection $\nabla$ and is closely related to the square of the Ray-Singer torsion. We refer the reader to [11, 12] for the precise definition of the form $\tau^{\mathrm{BH}}$ (see also [5, Section 3] for a short review). Using Proposition 4.2, we now can reformulate Theorem 1.6 of [5] as follows:

Theorem 4.1. Suppose $M$ is a closed oriented manifold of odd dimension $d=2 r-1$ and let $E$ be a complex vector bundle over $M$ endowed with a flat connection $\nabla$. Assume that there exists a symmetric bilinear form $b$ on $E$ so that the quadratic form (4.1) on $\operatorname{Det}\left(H^{\bullet}(M, E)\right)$ is defined. Then $\tau_{b, \nabla}^{\mathrm{BH}}= \pm \tau_{\nabla}$. 
Note that though the Burghelea-Haller form $\tau^{\mathrm{BH}}$ is defined only if $E$ admits a nondegenerate bilinear form $b$, the torsion quadratic form $\tau$ exists without this additional assumption. Therefore, $\tau$ can be viewed as an extension of $\tau^{\mathrm{BH}}$ to the case when the bilinear form $b$ does not exist.

\subsection{Relationship with the Farber-Turaev torsion}

The complex-valued combinatorial torsion has been introduced by Turaev [27-29] and, in a more general context, by Farber and Turaev $[18,19]$. The Farber-Turaev torsion depends on the Euler structure $\varepsilon$ and the orientation $\mathfrak{o}$ of the determinant line of the cohomology $H^{\bullet}(M, \mathbb{R})$ of $M$. The set of Euler structures $\operatorname{Eul}(M)$, introduced by Turaev, is an affine version of the integer homology $H_{1}(M, \mathbb{Z})$ of $M$. It has several equivalent descriptions $[9,13,27,28]$. For our purposes, it is convenient to adopt the definition from Section 6 of [28], where an Euler structure is defined as an equivalence class of nowhere vanishing vector fields on $M$ - see [28, Section 5] for the description of the equivalence relation. The Farber-Turaev torsion, depending on $\varepsilon, \mathfrak{o}$, and $\nabla$, is an element of the determinant line $\operatorname{Det}\left(H^{\bullet}(M, E)\right)$, which we denote by $\rho_{\varepsilon, 0}(\nabla)$.

Suppose $M$ is a closed oriented odd-dimensional manifold. Let $\varepsilon \in \operatorname{Eul}(M)$ be an Euler structure on $M$ represented by a nonvanishing vector field $X, \varepsilon=[X]$. Fix a Riemannian metric $g^{M}$ on $M$ and let $\Psi\left(g^{M}\right) \in \Omega^{d-1}(T M \backslash\{0\})$ denote the Mathai-Quillen form, [21, Section 7], [4, pp. 40-44]. Set

$$
\alpha_{\varepsilon}=\alpha_{\varepsilon}\left(g^{M}\right):=X^{*} \Psi\left(g^{M}\right) \in \Omega^{d-1}(M) .
$$

This is a closed differential form, whose cohomology class $\left[\alpha_{\varepsilon}\right] \in H^{d-1}(M, \mathbb{R})$ is closely related to the integer cohomology class, introduced by Turaev [28, Section 5.3] and called the characteristic class $c(\varepsilon) \in H_{1}(M, \mathbb{Z})$ associated with an Euler structure $\varepsilon$. More precisely, let PD : $H_{1}(M, \mathbb{Z}) \rightarrow H^{d-1}(M, \mathbb{Z})$ denote the Poincaré isomorphism. For $h \in H_{1}(M, \mathbb{Z})$, we denote by $\mathrm{PD}^{\prime}(h)$ the image of $\mathrm{PD}(h)$ in $H^{d-1}(M, \mathbb{R})$. Then

$$
\mathrm{PD}^{\prime}(C([X]))=-2\left[\alpha_{\varepsilon}\right]=-2\left[X^{*} \Psi\left(g^{M}\right)\right] .
$$

Burghelea and Haller made a conjecture, [11, Conjecture 5.1], relating the quadratic form $\tau_{b, \nabla}^{\mathrm{BH}}$ and $\rho_{\varepsilon, 0}(\nabla)$, which extends the Bismut-Zhang theorem [4]. In [5, Conjecture 1.12], we extended this conjecture to the case when $E$ does not admit a 
nondegenerate symmetric bilinear form. In view of Proposition 4.2, this conjecture can be reformulated as follows.

Following Farber [17], we denote by $\mathbf{A r g}_{\nabla}$ the unique cohomology class $\operatorname{Arg}_{\nabla} \in$ $H^{1}(M, \mathbb{C} / \mathbb{Z})$ such that for every closed curve $\gamma$ in $M$ we have

$$
\operatorname{det}\left(\operatorname{Mon}_{\nabla}(\gamma)\right)=\exp \left(2 \pi i\left\langle\operatorname{Arg}_{\nabla},[\gamma]\right\rangle\right)
$$

where $\operatorname{Mon}_{\nabla}(\gamma)$ denotes the monodromy of the flat connection $\nabla$ along the curve $\gamma$ and $\langle\cdot, \cdot\rangle$ denotes the natural pairing $H^{1}(M, \mathbb{C} / \mathbb{Z}) \times H_{1}(M, \mathbb{Z}) \rightarrow \mathbb{C} / \mathbb{Z}$.

Conjecture 4.5. Assume that $(E, \nabla)$ is a flat vector bundle over a closed odd-dimensional oriented manifold $M$. Then

$$
\tau_{\nabla}\left(\rho_{\varepsilon, \mathrm{o}}(\nabla)\right)=\mathrm{e}^{2 \pi i\left\langle\operatorname{Arg}_{\nabla}, c(\varepsilon)\right\rangle}
$$

The original Burghelea-Haller conjecture was proven independently by Burghelea-Haller [10] (up to sign) and Su-Zhang [26] (in full generality). Using this result, Theorem 4.1, and formula (1.12) of [5], we obtain the following theorem, which establishes Conjecture 4.5 up to sign in the case when $E$ admits a nondegenerate bilinear form:

Theorem 4.2. Suppose $M$ is a closed oriented manifold of odd dimension $d=2 r-1$ and let $E$ be a complex vector bundle over $M$ endowed with a flat connection $\nabla$. Assume that there exists a symmetric bilinear form $b$ on $E$. Then

$$
\tau_{\nabla}\left(\rho_{\varepsilon, \mathfrak{o}}(\nabla)\right)= \pm \mathrm{e}^{2 \pi i\left\langle\operatorname{Arg}_{\nabla}, c(\varepsilon)\right\rangle}
$$

Also, from Proposition 4.2 and Theorem 1.14 of [5], we obtain the following.

Theorem 4.3. (i) Under the same assumptions as in Conjecture 4.5, for each connected component $\mathcal{C}$ of the set Flat $(E)$ of flat connections on $E$ there exists a constant $R_{\mathcal{C}} \in \mathbb{C}$ with $\left|R_{\mathcal{C}}\right|=1$, such that

$$
\tau_{\nabla}\left(\rho_{\varepsilon, \mathfrak{o}}(\nabla)\right)=R_{\mathcal{C}} \cdot \mathrm{e}^{2 \pi i\left\langle\operatorname{Arg}_{\nabla}, c(\varepsilon)\right\rangle}, \quad \text { for all } \quad \nabla \in \mathcal{C} \text {. }
$$


(ii) If the connected component $\mathcal{C}$ contains an acyclic Hermitian connection then $R_{\mathcal{C}}=1$, i.e.

$$
\tau_{\nabla}\left(\rho_{\varepsilon, \mathrm{o}}(\nabla)\right)=\mathrm{e}^{2 \pi i\left\langle\operatorname{Arg}_{\nabla}, c(\varepsilon)\right\rangle}, \quad \text { for all } \quad \nabla \in \mathcal{C} .
$$

Note that the proof of Theorem 4.3 was obtained in [5] by much softer methods than those used in the proof of the original Burghelea-Haller conjecture $[10,26]$.

\section{The Cappell-Miller Torsion}

In this section, we first recall the definition of Cappell-Miller torsion

$$
T \in \operatorname{Det}\left(H^{\bullet}(M, E)\right) \otimes \operatorname{Det}\left(H^{\bullet}(M, E)\right)
$$

from [14], then establish its relationship with the torsion form $\tau$, and finally, under some additional assumptions, express $T$ in terms of the Farber-Turaev torsion $\rho_{\varepsilon, 0}$.

\subsection{The Cappell-Miller torsion of a finite-dimensional complex}

Let the complex $\left(C^{\bullet}, \partial\right)$ and the involution $\Gamma$ be as in Subsection 2.1. Recall that the element $\rho_{\Gamma} \in \operatorname{Det}\left(H^{\bullet}(\partial)\right)$ was introduced in (2.6).

In Section 5 of [14], Cappell and Miller introduced a torsion of a class of finitedimensional complexes, which in case of a complex of odd length $d=2 r-1$ and in the presence of the involution $\Gamma$, can be described as

$$
T=T_{\Gamma}:=\rho_{\Gamma} \otimes \rho_{\Gamma} \in \operatorname{Det}\left(H^{\bullet}(\partial)\right) \otimes \operatorname{Det}\left(H^{\bullet}(\partial)\right) .
$$

The torsion quadratic form $\tau_{\Gamma}$ defined in (2.3) can be viewed as an element of

$$
\operatorname{Det}\left(H^{\bullet}(\partial)\right)^{*} \otimes \operatorname{Det}\left(H^{\bullet}(\partial)\right)^{*} \simeq\left(\operatorname{Det}\left(H^{\bullet}(\partial)\right) \otimes \operatorname{Det}\left(H^{\bullet}(\partial)\right)\right)^{*} .
$$

It follows from (2.7) that $\tau_{\Gamma}$ is the dual of $T_{\Gamma}$, i.e.

$$
\tau_{\Gamma}\left(T_{\Gamma}\right)=1
$$


In particular, if the complex $\left(C^{\bullet}, \partial\right)$ is acyclic, then $T$ can be viewed as a complex number via the isomorphism $\operatorname{Det}\left(H^{\bullet}(\partial)\right) \simeq \mathbb{C}$, and in this case $T=1 / \tau$. It follows now from Lemma 2.7 that if the operator (2.9) is invertible, then

$$
T_{\Gamma}=\prod_{j=0}^{d} \operatorname{Det}\left(\left.\mathcal{B}^{2}\right|_{C^{j}}\right)^{(-1)^{j+1} j}
$$

Remark 5.2. In [14] the element $T$ is defined in slightly different terms. However, comparing the construction of $\rho_{\Gamma}$ with the construction of Section 5 of [14], one immediately sees that our definition coincides with the one of Cappell-Miller up to sign. To see that the signs agree one compares (5.3) with formula (5.43) of [14].

\subsection{The Cappell-Miller torsion of a flat vector bundle}

Let $E \rightarrow M$ be as in Section 3. Fix a Riemannian metric $g^{M}$ on $M$ and let $\Gamma$ denote the chirality operator (3.1). We shall use the notation introduced in Section 3. In particular, for each subset interval $\mathcal{J} \subset[0, \infty)$, we denote by $\Omega_{\mathcal{J}}^{j}(M, E)$ the image of $\Omega^{j}(M, E)$ under the spectral projection of $\left.\mathcal{B}^{2}\right|_{C^{j}}$ corresponding to the eigenvalues whose absolute value lie in J. Also $\mathcal{B}_{j, \mathcal{J}}$ denotes the restriction of $\mathcal{B}$ to $\Omega_{\mathcal{J}}^{j}(M, E)$ and $\Gamma_{\mathcal{J}}$ denotes the restriction of $\Gamma$ to $\Omega_{\mathcal{E}}^{\bullet}(M, E)$.

Fix $\lambda>0$ and let $T_{\Gamma_{[0, \lambda]}}$ be the Cappell-Miller torsion of the complex $\Omega_{[0, \lambda]}^{j}(M, E)$ corresponding to the chirality operator $\Gamma_{[0, \lambda]}$. Via the canonical isomorphism $H_{[0, \lambda]}^{\bullet}(M, E) \simeq$ $H^{\bullet}(M, E)$, we can view $T_{\Gamma_{[0, \lambda]}}$ as an element of $\operatorname{Det}\left(H^{\bullet}(\partial)\right) \otimes \operatorname{Det}\left(H^{\bullet}(\partial)\right)$.

Definition 5.4. Let $\theta \in(0,2 \pi)$ be an Agmon angle for the operator $\mathcal{B}_{(\lambda, \infty)}^{2}$. The CappellMiller torsion $T_{\nabla}$ of the flat vector bundle $(E, \nabla)$ over a closed oriented odd-dimensional manifold $M$ is the element

$$
T_{\nabla}:=\left[\prod_{j=0}^{d} \operatorname{Det}_{\theta}\left(\mathcal{B}_{(\lambda, \infty), j}^{2}\right)^{(-1)^{j+1} j}\right] \cdot T_{\Gamma_{[0, \lambda]}} \in \operatorname{Det}\left(H^{\bullet}(\partial)\right) \otimes \operatorname{Det}\left(H^{\bullet}(\partial)\right)
$$

It is shown in [14, Theorem 7.3] and also follows from Theorem 5.1 that $T_{\nabla}$ is independent of the choice of $\lambda$.

From (5.2), (5.4), and the definition (3.3) of $\tau_{\nabla}$, we obtain the following.

Theorem 5.1. $\tau_{\nabla}\left(T_{\nabla}\right)=1$. 
Hence, Conjecture 4.5 can be reformulated in the form

$$
T_{\nabla}=\mathrm{e}^{-2 \pi i\left\langle\operatorname{Arg}_{\nabla},(\varepsilon)\right\rangle} \cdot \rho_{\varepsilon, 0}(\nabla) \otimes \rho_{\varepsilon, 0}(\nabla) .
$$

Let $E^{*}$ denote the vector bundle dual to $E$. In particular, the fiber $E_{x}^{*}$ of $E^{*}$ at a point $x \in M$ is the dual vector space $E_{x}^{*}=\operatorname{Hom}_{\mathbb{C}}\left(E_{x}, \mathbb{C}\right)$. Let $\nabla^{*}$ denote the connection on $E^{*}$ dual to $\nabla$. Then the direct sum bundle $E \oplus E^{*}$ with the connection $\nabla \oplus \nabla^{*}$ is unimodular and its fibers have even dimension. Hence, cf., for example, Lemmas 3.2 and 3.3 of [19], the Reidemeister torsion

$$
\rho^{\mathrm{R}}\left(\nabla \oplus \nabla^{*}\right) \in \operatorname{Det}\left(H^{\bullet}\left(M, E \oplus E^{*}\right)\right) \simeq \operatorname{Det}\left(H^{\bullet}(M, E)\right) \otimes \operatorname{Det}\left(H^{\bullet}\left(M, E^{*}\right)\right) .
$$

is well defined and is equal to the Farber-Turaev torsion $\rho_{\varepsilon, 0}\left(\nabla \oplus \nabla^{*}\right)$. In particular, $\rho_{\varepsilon, \mathfrak{o}}\left(\nabla \oplus \nabla^{*}\right)$ is independent of $\varepsilon$ and $\mathfrak{o}$.

Farber and Turaev [19, p. 219] introduced the duality operator

$$
D: \operatorname{Det}\left(H^{\bullet}(M, E)\right) \rightarrow \operatorname{Det}\left(H^{\bullet}\left(M, E^{*}\right) .\right.
$$

Using the definition of the Poincaré-Reidemeister scalar product, cf. pp. 206 and 219 of [19] and Theorem 9.4 of [19], we obtain

$$
\rho_{\varepsilon, 0}(\nabla) \otimes D\left(\rho_{\varepsilon, 0}(\nabla)\right)=(-1)^{Z} \mathrm{e}^{2 \pi i\left\langle\operatorname{Arg}_{\nabla},(\varepsilon)\right\rangle} \cdot \rho^{\mathrm{R}}\left(\nabla \oplus \nabla^{*}\right),
$$

where $z \in \mathbb{N}$ is defined in formula (6.5) of [19]. Hence, (5.5) is equivalent to the following conjecture, originally made by Cappell and Miller [14]:

Conjecture 5.5 (Cappell-Miller). Assume that $(E, \nabla)$ is a flat vector bundle over a closed odd-dimensional oriented manifold $M$. Then the Cappell-Miller torsion is related to the Reidemeister torsion by the equation

$$
(1 \otimes D) T_{\nabla}=(-1)^{z} \rho^{\mathrm{R}}\left(\nabla \oplus \nabla^{*}\right),
$$

where $z \in \mathbb{N}$ is defined in formula (6.5) of [19] (The sign factor $(-1)^{z}$ is missing in [14] because of a different sign convention.) 
Theorems 4.2 and 4.3 give a partial solution of this conjecture. In particular, Theorem 4.3 says that Conjecture 5.5 holds up to the factor $R_{C}$ and holds exactly in the case when $\nabla$ belongs to a connected component of the space Flat $(E)$, which contains an acyclic Hermitian connection. Theorem 4.2 states that Conjecture 5.5 holds up to sign if $E$ admits a nondegenerate bilinear form $b$.

\section{Acknowledgments}

We would like to thank Sylvain Cappell for his comments on an earlier version of this paper and informing us about the revised preprint [14]. The first author was supported in part by the NSF grant DMS-0706837. The second author was supported in part by the Swiss National Science foundation, the programme SPECT, and the European Community through the FP6 Marie Curie RTN ENIGMA (MRTN-CT-2004-5652).

\section{References}

[1] Atiyah, M. F., V. K. Patodi, and I. M. Singer. "Spectral asymmetry and Riemannian geometry." Mathematical Proceedings of the Cambridge Philosophical Society 77, no. 1 (1975): 43-69.

[2] Atiyah, M. F., V. K. Patodi, and I. M. Singer. "Spectral asymmetry and Riemannian geometry 2." Mathematical Proceedings of the Cambridge Philosophical Society 78, no. 3 (1975): 405-32.

[3] Berline, N., E. Getzler, and M. Vergne. Heat Kernels and Dirac Operators. Grundlehren der Mathematischen Wissenschaften [Fundamental Principles of Mathematical Sciences], vol. 298. Berlin: Springer, 1992.

[4] Bismut, J.-M., and W. Zhang. "An extension of a theorem by Cheeger and Müller." Astérisque 205 (1992).

[5] Braverman, M., and T. Kappeler. "Comparison of the refined analytic and the BurgheleaHaller torsions." Annales de l'Institute Fourier (Grenoble) 57, no. 7 (2007): 2361-87.

[6] Braverman, M., and T. Kappeler. "Ray-Singer type theorem for the refined analytic torsion." Journal of Functional Analysis 243 (2007): 232-56.

[7] Braverman, M., and T. Kappeler. "Refined analytic torsion as an element of the determinant line." Geometry \& Topology 11 (2007): 139-213.

[8] Braverman, M., and T. Kappeler. "Refined analytic torsion." Journal of Differential Geometry 78, no. 1 (2008): 193-267.

[9] Burghelea, D. "Removing metric anomalies from Ray-Singer torsion." Letters in Mathematical Physics 47 (1999): 149-58.

[10] Burghelea, D., and S. Haller. "Complex valued Ray-Singer torsion 2." (2006): preprint arXiv:math.DG/0610875.

[11] Burghelea, D., and S. Haller. “Complex valued Ray-Singer torsion." J. Funct. Anal. 248, no. 1 (2007): 27-78. 
[12] Burghelea, D., and S. Haller. "Torsion, as a function on the space of representations." (2005): preprint arXiv:math.DG/0507587.

[13] Burghelea, D., and S. Haller. "Euler structures, the variety of representations and the MilnorTuraev torsion." Geometry \& Topology 10 (2006): 1185-1238.

[14] Cappell, S. E., and E. Y. Miller. "Analytic torsion for flat bundles and holomorphic bundles with $(1,1)$ connections." (2007): preprint arXiv:math.DG/0710123v1.

[15] Cheeger, J. "Analytic torsion and the heat equation." Annals of Mathematics 109 (1979): 259-300.

[16] Farber, M. "Combinatorial invariants computing the Ray-Singer analytic torsion." Differential Geometry and its Applications 6 (1996): 351-66.

[17] Farber, M. "Absolute torsion and eta-invariant." Mathematische Zeitschrift 234, no. 2 (2000): 339-49.

[18] Farber, M., and V. Turaev. "Absolute Torsion." In Rothenberg Festschrift (1998), 73-85. Contemporary Mathematics 231. Tel Aviv Topology Conference. Providence, RI: American Mathematical Society, 1999.

[19] Farber, M., and V. Turaev. "Poincaré-Reidemeister metric, Euler structures, and torsion." Journal für die Reine und Angewandte Mathematik 520 (2000): 195-225.

[20] Gilkey, P. B. "The Eta Invariant and Secondary Characteristic Classes of Locally Flat Bundles." In Algebraic and Differential Topology-Global Differential Geometry. Teubner-Texte zur Mathematik 70. Leipzig: Teubner, 1984, pp. 49-87.

[21] Mathai, V., and D. Quillen. "Superconnections, Thom classes, and equivariant differential forms." Topology 25 (1986): 85-110.

[22] Milnor, J. "Whitehead torsion." Bulletin of the American Mathematical Society 72 (1966): 358-426.

[23] Müller, W. "Analytic torsion and R-torsion of Riemannian manifolds." Advances in Mathematics 28 (1978): 233-305.

[24] Ray, D. B., and I. M. Singer. "R-torsion and the Laplacian on Riemannian manifolds." $A d$ vances in Mathematics 7 (1971): 145-210.

[25] Shubin, M. A. Pseudodifferential Operators and Spectral Theory. Berlin, New York: Springer, 1987.

[26] Su, G., and W. Zhang. "A Cheeger-Mueller theorem for symmetric bilinear torsions." (2006): preprint arXiv:math.DG/0610577.

[27] Turaev, V. G. "Reidemeister torsion in knot theory." Russian Mathematical Surveys 41 (1986): 119-82.

[28] Turaev, V. G. "Euler structures, nonsingular vector fields, and Reidemeister-type torsions." Mathematics of the USSR-Izvestiya 34 (1990): 627-62.

[29] Turaev, V. G. Introduction to Combinatorial Torsions. Notes taken by Felix Schlenk. Lectures in Mathematics, ETH Zürich. Basel: Birkhäuser, 2001. 